\title{
Downregulation of MACC1 inhibits invasion, migration and proliferation, attenuates cisplatin resistance and induces apoptosis in tongue squamous cell carcinoma
}

\author{
HAI-FENG LI $^{1 *}$, YE-QING LIU ${ }^{1 *}$, ZHUO-JIAN SHEN ${ }^{3}$, XIANG-FENG GAN ${ }^{3}$, JING-JING HAN ${ }^{1}$, \\ YUN-YUN LIU ${ }^{4}$, HAI-GANG LI $^{1}$ and ZHI-QUAN HUANG ${ }^{2}$ \\ Departments of ${ }^{1}$ Pathology, ${ }^{2}$ Oral and Maxillofacial Surgery, ${ }^{3}$ Cardio-Thoracic Surgery, ${ }^{4}$ Gynecology, \\ Sun Yat-Sen Memorial Hospital, Sun Yat-Sen University, Guangzhou 510120, P.R. China
}

Received September 22, 2014; Accepted October 21, 2014

DOI: 10.3892/or.2014.3612

\begin{abstract}
The aim of the present study was to investigate the expression and function of metastasis-associated in colon cancer 1 (MACC1) in tongue squamous cell carcinoma (TSCC) and its relationship with the expression of extracellular matrix metalloproteinase inducer (EMMPRIN) (CD147). Levels of MACC1 and EMMPRIN expression were analyzed in 65 paraffin-embedded tissue specimens of TSCC and in the adjacent non-cancerous tissues using immunohistochemistry (IHC). MACC1 expression was highly associated with lymphatic metastasis and EMMPRIN expression. Overexpression of MACC1 was significantly correlated with poor overall patient survival. A small interfering RNA (siRNA) was delivered into TSCCA cells to downregulate MACC1 expression. The CCK-8 assay showed that TSCCA cell proliferation was markedly reduced and that cisplatin resistance was attenuated. The suppression of MACC1 promoted the apoptosis of the TSCCA cell line. A Transwell experiment demonstrated that the migration and invasion abilities of the
\end{abstract}

Correspondence to: Professor Hai-Gang Li, Department of Pathology, Sun Yat-Sen Memorial Hospital, Sun Yat-Sen University, 107 Yanjiang Road, Guangzhou 510120, P.R. China

E-mail: 13728089120@126.com

Professor Zhi-Quan Huang, Department of Oral and Maxillofacial Surgery, Sun Yat-Sen Memorial Hospital, Sun Yat-Sen University, 107 Yanjiang Road, Guangzhou 510120, P.R. China

E-mail: zhiquanhuang1978@126.com

*Contributed equally

Abbreviations: TSCC, tongue squamous cell carcinoma; MACC1, metastasis-associated in colon cancer; EMMPRIN, extracellular matrix metalloproteinase inducer; MACC1-siRNA, MACC1 small interfering RNA; IHC, immunohistochemistry; HNSCC, head and neck squamous cell carcinoma; OSCC, oral squamous cell carcinoma

Key words: tongue squamous cell carcinoma, MACC1, EMMPRIN, invasion, migration, uPA, MMP2
TSCCA cells were extremely downregulated. An ELISA experiment and western blotting revealed that the secretion of urokinase-type plasminogen activator system (uPA) in the supernatant of the culture medium and UPA expression were significantly reduced. Experiments revealed that the secretion of metalloproteinase 2 (MMP2) in the supernatant of the culture medium and MMP2 expression were not affected. $\mathrm{MACC1}$ may serve as a novel biomarker and therapeutic target for TSCC.

\section{Introduction}

Head and neck squamous cell carcinoma (HNSCC) is the sixth most common cancer worldwide. In China, statistical data reveal that the incidence of HNSCC in males and females is 8.17 and 4.6/100,000 individuals, respectively, and HNSCC accounts for 5.1 and $2.7 \%$ of all malignant tumors each year. Statistically, oral squamous cell carcinoma (OSCC) is the most common HNSCC and accounts for 2-3\% for all malignant tumors. Tongue squamous cell carcinoma (TSCC) is the most common carcinoma among OSCC cases, accounting for $25-40 \%$ of mouth neoplasms (1). The incidence of TSCC has increased in recent years, making it a major public health concern. In spite of the wide use of combination therapy, such as surgery-radio-chemotherapy, the mortality rate of TSCC remains high. The 5-year survival rate of TSCC patients is less than $50 \%$ (2). To our knowledge, the primary cause of therapy failure is recurrence in situ and distant metastases. Therefore, it is important to identify new biomarkers for therapeutic targets.

The metastasis-associated in colon cancer 1 (MACC1) gene was identified in a genome-wide screen of human colon cancer, and its expression is closely related to the metastasis of colon cancer (3). MACC1 is a key regulator of the hepatocyte growth factor (HGF)-MET signaling pathway, which is related to colon cancer metastasis $(4,5)$. MACC1 downregulation inhibits tumorigenicity through the $\mathrm{Akt} / \beta$-catenin signaling pathway in nasopharyngeal carcinoma cells (6). Recently, many studies have reported that MACC1 is involved in cancer development and progression by promoting tumor cell proliferation, migration and invasion. Further studies 
have demonstrated that MACC1 upregulates cell growth and metastatic progression in several solid tumors, including lung cancer $(7,8)$, hepatocellular carcinoma (4,9-11), ovarian carcinoma (12), pancreatic cancer (13), breast cancer (14) and colon cancer $(4,15,16)$. To our knowledge, no research regarding the function of $\mathrm{MACC} 1$ in TSCC has been reported. In the present study, we determined that the overexpression of MACC1 was significantly correlated with the poor overall survival of the studied patients. The knockdown of MACC1 inhibited cell proliferation, migration and invasion, the knockdown of MACC1 also attenuated cisplatin resistance in TSCCA cells and contributed to apoptosis. Furthermore, we demonstrated that MACC1 influences the invasion and migration of TSCCA cells by secreting and activating urokinase-type plasminogen activator system (uPA) but not metalloproteinase 2 (MMP2).

\section{Materials and methods}

Cell culture. TSCCA cells were purchased from the College of Stomatology, Shanghai Jiao Tong University (Shanghai, China) and cultured in RPMI-1640 medium (Gibco, Carlsbad, CA, USA) containing $10 \%$ fetal bovine serum (Biological Industries, Israel) at $37^{\circ} \mathrm{C}$ with $5 \% \mathrm{CO}_{2}$.

Small interfering RNA (siRNA) transfection. siRNA duplexes were synthesized and purified by GenePharma (Shanghai). The siRNA sequences for MACC1 were as follows: sense, 5'-AAGAUUGGACUUGUACACUGCTT-3' and antisense, 5'-TTUUCUAACCUGAACAUGUGACG-3'. The transfection of MACC1-siRNA was performed using the Lipofectamine 2000 reagent (Invitrogen, Carlsbad, CA, USA) following the manufacturer's instructions. The transfection of MACC1-NC was conducted in the same manner to serve as the control. In this study, the experimental group was transfected with MACC1-siRNA, whereas the control group was transfected with MACC1-NC.

Immunohistochemistry (IHC). Sixty-five paraffin-embedded specimens of TSCC and the adjacent non-cancerous tissues were collected from 1996 to 2006 at the Department of Oral and Maxillofacial Surgery, Sun Yat-Sen Memorial Hospital. The study was approved by the Institutional Research Ethics Committee of Sun Yat-Sen Memorial Hospital of Sun Yat-Sen University. Consent was obtained from the patients, and the clinical research materials were collected from the patients prior to clinical treatment. Immunohistochemical staining was performed with a polyclonal anti-MACC1 antibody (Abcam Ltd., Hong Kong) and a polyclonal anti-extracellular matrix metalloproteinase inducer (EMMPRIN) antibody (Abgent, Suzhou, China) according to standard protocols. Briefly, the tissue sections were blocked sequentially with $3 \% \mathrm{H}_{2} \mathrm{O}_{2}$ and normal serum and then incubated with MACC1 (1:100) and EMMPRIN (1:100) at $4^{\circ} \mathrm{C}$ overnight. The tissue sections were incubated with a biotinylated secondary antibody and conjugated with a streptavidin-HRP complex (ready to use SP kit; Zhongshan Co., Beijing, China). Finally, the sections were visualized with 3-3'-diaminobenzidine and counterstained with hematoxylin. Sections of human colon tissues stained positive with these antibodies were used as the positive control for MACC1, TSCC tissue known to have positive staining for
EMMPRIN were used as a positive control, and PBS was used instead of the primary antibodies for the negative controls.

Evaluation of IHC staining. The IHC evaluation of the tissues was conducted by 2 pathologists (H-F.L. and H-G.L.) who assessed the number of positive cells and the intensity of staining. The positive results were judged by semi-quantitative points. The staining intensity scores were 0 (negative), 1 (weak), 2 (medium) and 3 (strong). The integral of the rate of positive cells was $0(0 \%), 1(1-25 \%), 2(26-50 \%)$ and $3(>50 \%)$. The staining intensity score and the proportional score were added to obtain the total score (17). A total score $\geq 3$ was considered to represent high MACC1 expression. A total score $<3$ was considered to represent low MACC1 expression. For the EMMPRIN staining evaluation, samples in which $>10 \%$ of tumor cells were stained positive with anti-EMMPRIN antibodies were evaluated as strongly positive $(++), \leq 10 \%$ cells were positive (+) and no detectable staining was negative (-) (2). The clinical features of the patients are summarized in Table I, and the overall survival time of the patients is analyzed in Fig. 2.

Western blot analysis. After transfection, the cells were collected, and the total protein was extracted from the cells. Thirty microliters of protein was loaded and separated using $10 \%$ sodium dodecyl sulfate polyacrylamide gel electrophoresis (SDS-PAGE) (Beyotime, Shanghai, China) and transferred to Immobilon-P transfer membranes (PVDF) (Beyotime). The membranes were blocked with 5\% non-fat milk in Tris-buffered saline (TBS) containing $0.1 \%$ Tween-20 for $1 \mathrm{~h}$ at room temperature. The blots were probed with the relevant primary antibodies overnight at $4^{\circ} \mathrm{C}$, washed in TBST and probed with a species-specific horseradish peroxidase-conjugated secondary antibody (anti-rabbit; Shanghai ExCell Biology, Inc., China). The anti-MACC1 antibody (Abcam), anti-uPA antibody (GeneTex, Wuhan, China) and the anti-MMP2 antibody (GeneTex) were used to probe the alterations of the protein. GAPDH (KangCheng-Biotech, Shanghai, China) was used as a loading control. The western blot analysis process was performed according to standard protocols.

CCK-8 assays. After $24 \mathrm{~h}$ of transfection, the cells were collected, and $5 \times 10^{3}$ cells/well were plated into 96 -well plates. All of the cells were incubated overnight. After 48, 72 and $96 \mathrm{~h}$ of transfection, the cell proliferation was determined by the Cell Counting Kit-8 (Dojindo, Kyushu, Japan). The medium of each well was removed and a mixture of $10 \mu \mathrm{l}$ CCK-8 and $90 \mu 1$ of $10 \%$ FBS RPMI-1640 was added. The plates were incubated for a further $3 \mathrm{~h}$, and the absorbance at $450 \mathrm{~nm}$ was measured by a microplate reader (Multiskan MK3; Thermo Electric, Shanghai, China). All of the groups at each time-point had 5 wells. The relative cell survival (\%) was determined by the following formula: $\left(\mathrm{OD}_{\text {siRNA }} / \mathrm{OD}_{\text {scramble }}\right) \times 100 \%$.

After $24 \mathrm{~h}$ of transfection, the TSCCA cells were seeded at a density of $1 \times 10^{4}$ cells/well into 96 -well plates and incubated overnight. Six concentrations of cisplatin $(0,2.5,5,10,20$ and $40 \mu \mathrm{mol})$ were added into each well with equal volume. After $48 \mathrm{~h}$, the cell proliferation was determined using the CCK-8. The medium from each well was removed, and a mixture of $10 \mu \mathrm{l}$ CCK-8 and $90 \mu \mathrm{l}$ of $10 \%$ FBS RPMI-1640 was added. 
Table I. Association of MACC1 expression with the clinicopathological features of the tongue squamous cell carcinoma patients.

\begin{tabular}{|c|c|c|c|}
\hline \multirow[b]{2}{*}{ Characteristics } & \multicolumn{2}{|c|}{ MACC 1 expression } & \multirow[b]{2}{*}{ P-value } \\
\hline & Low $(\%)$ & High (\%) & \\
\hline \multicolumn{4}{|l|}{ Age (years) } \\
\hline$\leq 40$ & $7(53.8)$ & $6(46.2)$ & 0.615 \\
\hline$>40$ & $32(61.5)$ & $20(38.5)$ & \\
\hline \multicolumn{4}{|l|}{ Gender } \\
\hline Male & $22(53.7)$ & $19(46.3)$ & 0.176 \\
\hline Female & $17(70.8)$ & $7(29.2)$ & \\
\hline \multicolumn{4}{|l|}{ Differentiation } \\
\hline Well & $8(66.7)$ & $4(33.3)$ & 0.553 \\
\hline Moderate & $29(58.0)$ & $21(42.0)$ & \\
\hline Poor & $2(66.7)$ & $1(33.3)$ & \\
\hline \multicolumn{4}{|c|}{ Lymphatic metastasis } \\
\hline No & $34(70.8)$ & $14(21.2)$ & 0.003 \\
\hline Yes & $5(29.4)$ & $12(70.6)$ & \\
\hline \multicolumn{4}{|l|}{ Clinical stage } \\
\hline I & $13(86.7)$ & $2(13.3)$ & 0.088 \\
\hline II & $14(51.9)$ & $13(48.1)$ & \\
\hline III & $4(44.4)$ & $5(55.6)$ & \\
\hline IV & $8(57.1)$ & $6(42.9)$ & \\
\hline \multicolumn{4}{|l|}{ EMMPRIN } \\
\hline$(-)$ & $17(85.0)$ & $3(15.0)$ & 0.003 \\
\hline$(+)$ & $10(58.8)$ & $7(41.2)$ & \\
\hline$(++)$ & $12(42.9)$ & $16(57.1)$ & \\
\hline
\end{tabular}

MACC1, metastasis-associated in colon cancer 1; TSCC, tongue squamous cell carcinoma; EMMPRIN, extracellular matrix metalloproteinase inducer.

The plates were incubated for a further $3 \mathrm{~h}$, and the absorbance at $450 \mathrm{~nm}$ was measured by a microplate reader (Multiskan MK3; Thermo Electric). All of the groups treated with each concentration had 5 wells. The relative cell survival (\%) was determined by the following formula $\left(\mathrm{OD}_{\text {siRNA }} / \mathrm{OD}_{\text {scramble }}\right) \mathrm{x}$ $100 \%$.

Apoptosis assays. Cells were seeded at a density of 5x10 $/$ well in 6 -well plates. The experimental group and the control group each had 3 wells. After incubating overnight, the experimental and the control groups of TSCCA cells were transfected with MACC1-NC and MACC1-siRNA, respectively. After $24 \mathrm{~h}$ of transfection, $5 \mu \mathrm{mol}$ cisplatin was added to each well to induce apoptosis; the plates were then incubated for $48 \mathrm{~h}$. The TSCCA cells were collected, including the supernatant of the culture medium, and the total cells in each well were washed with PBS twice, and then $300 \mu 11 \mathrm{X}$ buffer diluents was added to each sample. A total of $300 \mu \mathrm{l}$ of the cell suspension was divided into $200 \mu \mathrm{l}$ for the apoptosis analysis group and $100 \mu \mathrm{l}$ for the loading control. The former group was incubated with $2.5 \mu \mathrm{l}$ of 7-AAD and $2.5 \mu \mathrm{l}$ of PE (both from BD Biosciences, Franklin Lakes, NJ, USA) antibodies for $15 \mathrm{~min}$ at room temperature in the dark. All of the samples were analyzed for apoptosis using the Becton-Dickinson FACSVerse ${ }^{\mathrm{TM}}$ (BD Biosciences).

In vitro migration and invasion assays. For the Transwell migration assays, $3 \times 10^{5}$ cells were seeded into the upper chamber of a polycarbonate Transwell plate (Corning, Suzhou, China). The lower chamber of the Transwell plate contained $10 \%$ FBS RPMI-1640. The cells were incubated at $37^{\circ} \mathrm{C}$ with $5 \% \mathrm{CO}_{2}$ for $18 \mathrm{~h}$. For the Transwell invasion assays, we mixed $10 \mu \mathrm{l}$ of Matrigel basement membrane matrix (BD Biosciences) and $90 \mu \mathrm{l}$ of cold serum-free RPMI-1640 per well and then placed the mixture into the upper chamber of the Transwell. After 4-6 h of incubation at $37^{\circ} \mathrm{C}$ with $5 \% \mathrm{CO}_{2}, 1 \times 10^{5}$ cells were seeded into the upper chamber of the polycarbonate Transwell plate; the lower chamber of the Transwell plate contained 20\% FBS RPMI-1640. The cells were incubated at $37^{\circ} \mathrm{C}$ with $5 \% \mathrm{CO}_{2}$ for $48 \mathrm{~h}$. Subsequently, the cells inside of the upper chamber were removed by cotton swabs. Migratory and invasive cells on the lower chamber surface were fixed in $4 \%$ formaldehyde, stained with $0.5 \%$ crystal violet and counted (3 random x 40 fields/well). The same experiments were performed a minimum of 3 times.

ELISA assays. Sample preparation. After $24 \mathrm{~h}$ of transfection and incubation in 6-well plates with MACC1-NC and MACC1-siRNA, respectively, the supernatant of the culture medium of the TSCCA cells was collected and centrifuged at $1,000 \mathrm{x}$ for $20 \mathrm{~min}$ at room temperature. The uPA and MMP2 concentrations in the supernatant of the culture medium were quantified using a uPA ELISA kit (Cloud-Clone Corp., Houston, TX, USA) and an MMP2 ELISA kit (ExCell Biology Inc.) according to the manufacturers' recommended protocols. The sample analyses were executed in triplicate and were repeated a minimum of 3 times.

Statistical analysis. All of the statistical analyses were conducted using SPSS 13.0 statistical software. The Wilcoxon X, Mann-Whitney U-test and Kruskal-Wallis analyses were used to analyze the correlation between MACC1 expression and the clinicopathological characteristics. The relationship between MACC1 and EMMPRIN expression was assessed by the Spearman method. The survival curves were plotted using the Kaplan-Meier method and were compared with the log-rank test. Student's t-test was used to compare the levels of cell proliferation, cisplatin resistance, migration and invasion ability between the different groups. $\mathrm{P}<0.05$ was considered to indicate a statistically significant result.

\section{Results}

MACCl overexpression is associated with lymphatic metastasis and EMMPRIN expression and contributes to poor overall survival in TSCC. Sixty-five paraffin-embedded specimens of TSCC and their adjacent non-cancerous tissues were examined by IHC. The results showed that MACC1 expression in TSCC was significantly higher than that in the non-cancerous epithelial tissue adjacent to the carcinoma. 


\section{EMMPRIN expression}
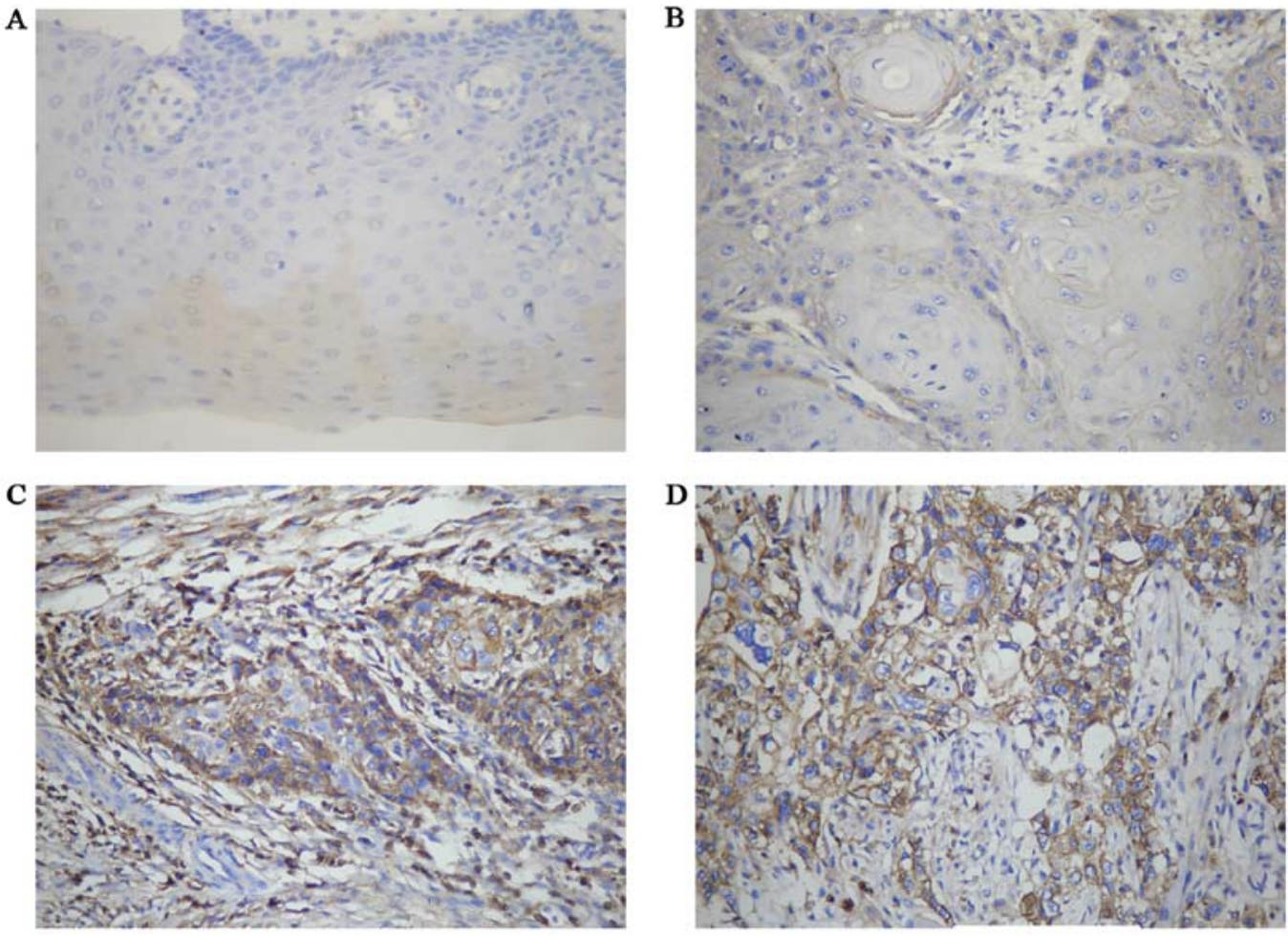

\section{MACC1 expression}
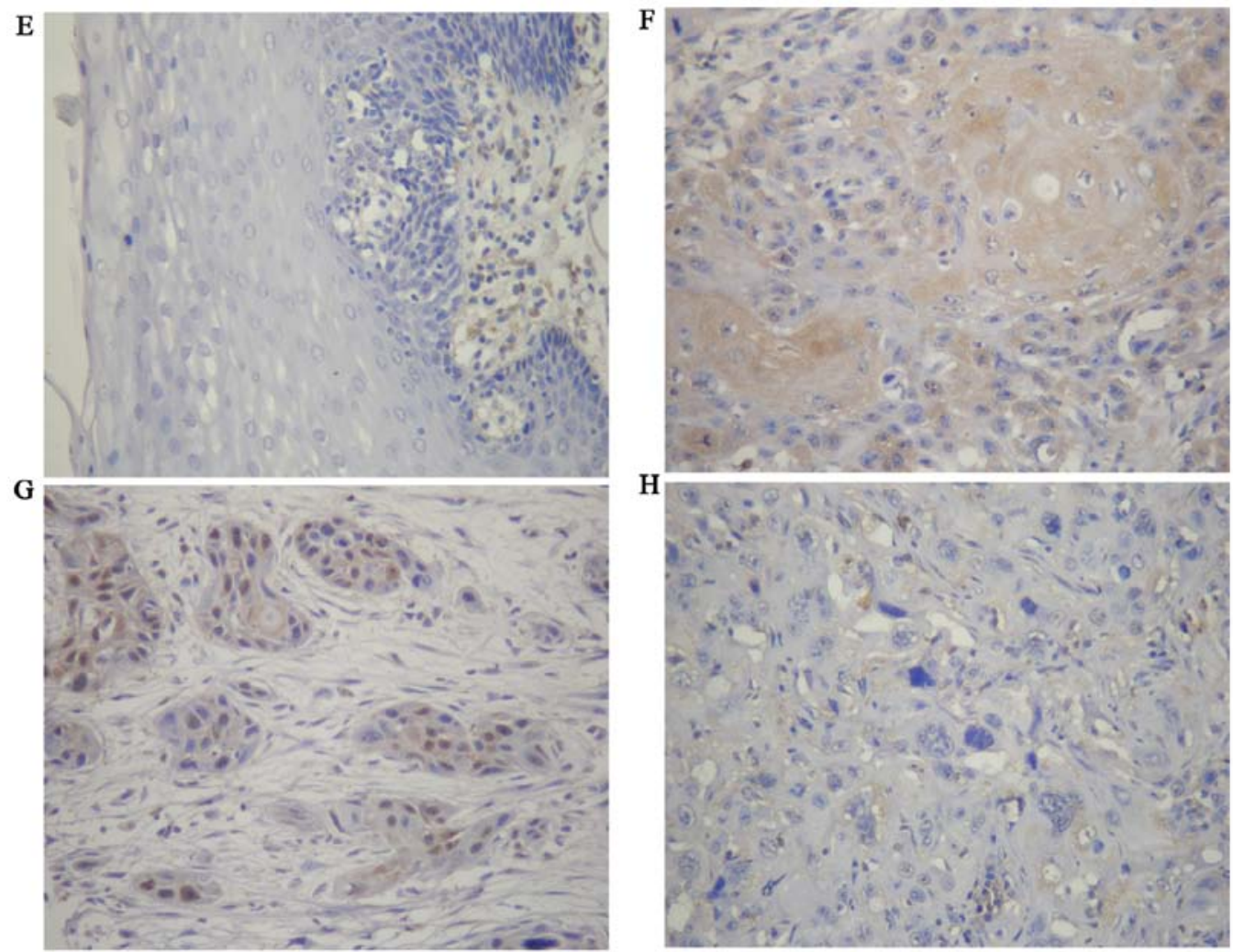

Figure 1. Immunohistochemical analysis of MACC1 and EMMPRIN expression in TSCC and in the adjacent non-cancerous epithelial tissues. (A) Negative EMMPRIN expression in non-cancerous epithelium adjacent to carcinoma of the tongue. (B) Positive EMMPRIN expression in a well-differentiated TSCC (+). (C) Strongly positive EMMPRIN expression in a moderately differentiated TSCC (++). (D) Strongly positive EMMPRIN expression in a poorly differentiated TSCC (++). (E) Negative MACC1 expression in non-cancerous epithelium adjacent to carcinoma of the tongue. (F) High MACC1 expression in a well-differentiated TSCC. (G) High MACC1 expression in a moderately differentiated TSCC. (H) Low MACC1 expression in a poorly differentiated TSCC. The MACC1 and EMMPRIN expression levels were significantly higher in TSCC than that in the non-cancerous epithelial tissues adjacent to the carcinoma $(\mathrm{P}<0.05)$; original magnification $\mathrm{x} 200$. MACC1, metastasis-associated in colon cancer 1; TSCC, tongue squamous cell carcinoma; EMMPRIN, extracellular matrix metalloproteinase inducer. 
Table II. Multivariate analysis of prognostic factors associated with survival time in the 68 patients with TSCC.

\begin{tabular}{lccccccc}
\hline Variable & B & S.E & Wald & Df & Sig & R & Exp (B) \\
\hline Gender & 0.746 & 0.461 & 2.618 & 1 & 0.106 & 0.053 & 2.109 \\
Age (years) & -0.694 & 0.434 & 2.561 & 1 & 0.110 & -0.050 & 0.500 \\
Differentiation & -0.063 & 0.354 & 0.037 & 1 & 0.847 & 0.000 & 0.934 \\
Lymphatic metastasis & 0.102 & 0.512 & 0.040 & 1 & 0.842 & 0.000 & 1.1073 \\
Clinical stage & 0.205 & 0.248 & 0.681 & 1 & 0.409 & 0.0171 & 1.227 \\
EMMPRIN expression & 0.987 & 0.345 & 8.188 & 1 & $\mathbf{0 . 0 0 4 2}$ & 0.167 & 2.684 \\
MACC1 expression & 1.888 & 0.503 & 14.072 & 1 & $\mathbf{0 . 0 0 0 2}$ & 0.233 & 6.606
\end{tabular}

TSCC, tongue squamous cell carcinoma; MACC1, metastasis-associated in colon cancer 1; EMMPRIN, extracellular matrix metalloproteinase inducer.

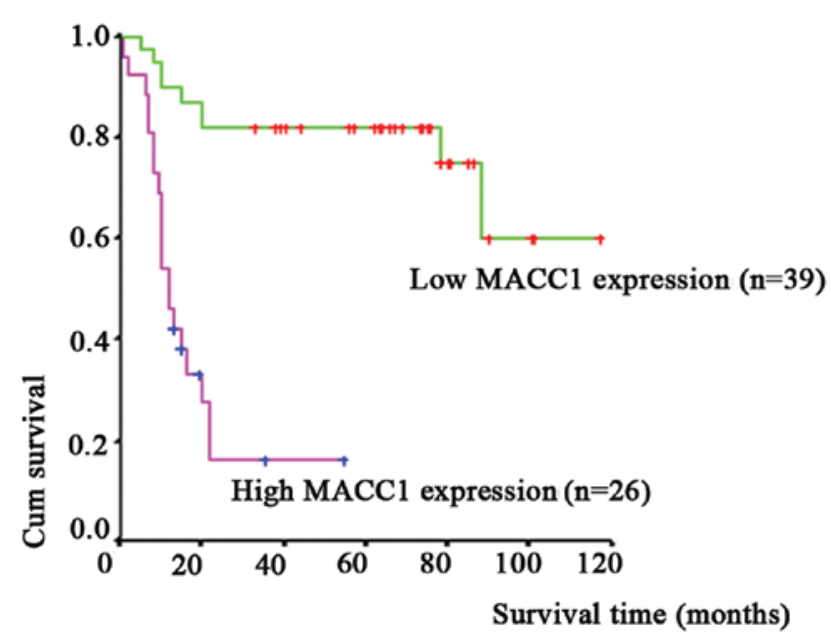

Figure 2. Kaplan-Meier estimates of the overall survival time of patients with high MACC1 expression vs. patients with low MACC1 expression. The patients with high MACC1 expression demonstrated a significantly shorter overall survival time $(\mathrm{P}<0.05)$. MACC1, metastasis-associated in colon cancer 1 .

MACC1-positive products were mainly localized in the cytoplasm of TSCC. The overexpression of MACC1 was closely associated with lymphatic metastasis and EMMPRIN expression ( $\mathrm{P}=0.003, \mathrm{P}=0.003)$. Our previous study demonstrated that EMMPRIN expression was localized in the cytomembrane and its expression was significantly associated with tumor diameter and poor survival (2). No significant association was found between MACC1 overexpression and other clinicopathological characteristics, including age, gender and differentiation (Fig. 1 and Table I). We did not find a significant difference between MACC1 expression and clinical stage (Table I, $\mathrm{P}=0.088$ ), however, the data demonstrated a positive trend. High MACC1 expression levels tended to occur often in patients with advanced stages (III+IV) of TSCC. We suggest that the unclear relationship between the high expression of MACC1 and the clinical stage may be elucidated if we include more cases in our further studies. Furthermore, we followed up the patients who were diagnosed with TSCC in this study. We found that the patients with MACC1 overexpression were often suffering from distant metastasis and were approaching death. The survival curves were generated using the Kaplan-Meier method and were compared with the logrank test. Multivariate analysis demonstrated that MACC1 and EMMPRIN (CD147) overexpression were closely related with the overall survival time of the patients (Table II, $\mathrm{P}=0.0002$, $\mathrm{P}=0.0042$ ) respectively. Thirty-nine patients with low MACC1 expression levels were followed up from 1 to 117 months, with a median overall survival of 66 months. Twenty-six patients with high MACC1 expression levels were followed up from 1 to 55 months, with a median overall survival of 12 months. Conclusively, the patients with high MACC1 expression levels demonstrated a significantly shorter survival time (Fig. 2 and Table II, $\mathrm{P}<0.05)$.

Downregulation of MACC1 significantly inhibits cell proliferation, attenuates cisplatin resistance and induces apoptosis of TSCC in vitro. The western blot analysis showed that the knockdown of MACC1 resulted in the inhibition of MACC1 protein expression in TSCCA cells (Fig. 3G). We tested three time-points (48, 72 and $96 \mathrm{~h}$ ) after the knockdown of MACC1. The results of the cell proliferation tested by CCK-8 showed that MACC1-siRNA-transfected TSCCA cells exhibited decreased cell growth ability $(\mathrm{P}<0.05)$ compared with the control group (Fig. $3 \mathrm{~A}-\mathrm{C}, \mathrm{P}<0.05$ ). The inhibition rate of MACC1 knockdown on proliferation was $25 \%$. Drug resistance to cisplatin assays revealed that MACC1-siRNAtransfected TSCCA cells had attenuated drug resistance to cisplatin, especially at the concentrations of 10 and $5 \mu \mathrm{mol}$ cisplatin (Fig. 3D and E, $\mathrm{P}<0.05$ ). The apoptosis assay showed that after transfection with MACC1-siRNA, the early apoptosis rate increased from $8.45 \pm 1.30$ to $12.66 \pm 1.29 \%$. The difference between the 2 groups was statistically significant (Fig. 3F, P<0.05). Our studies demonstrated that the downregulation of MACC1 significantly inhibited proliferation, attenuated cisplatin resistance and induced apoptosis in the TSCCA cells in vitro.

Downregulation of MACCl contributes to the inhibition of migration and invasion of TSCC in vitro. The Transwell penetration assay of migration demonstrated that the number of migratory cells transfected with MACC1-NC and MACC1-siRNA at $18 \mathrm{~h}$ was $837 \pm 31$ and $532 \pm 36 /$ field of view, respectively. The Transwell penetration assay of invasion had 

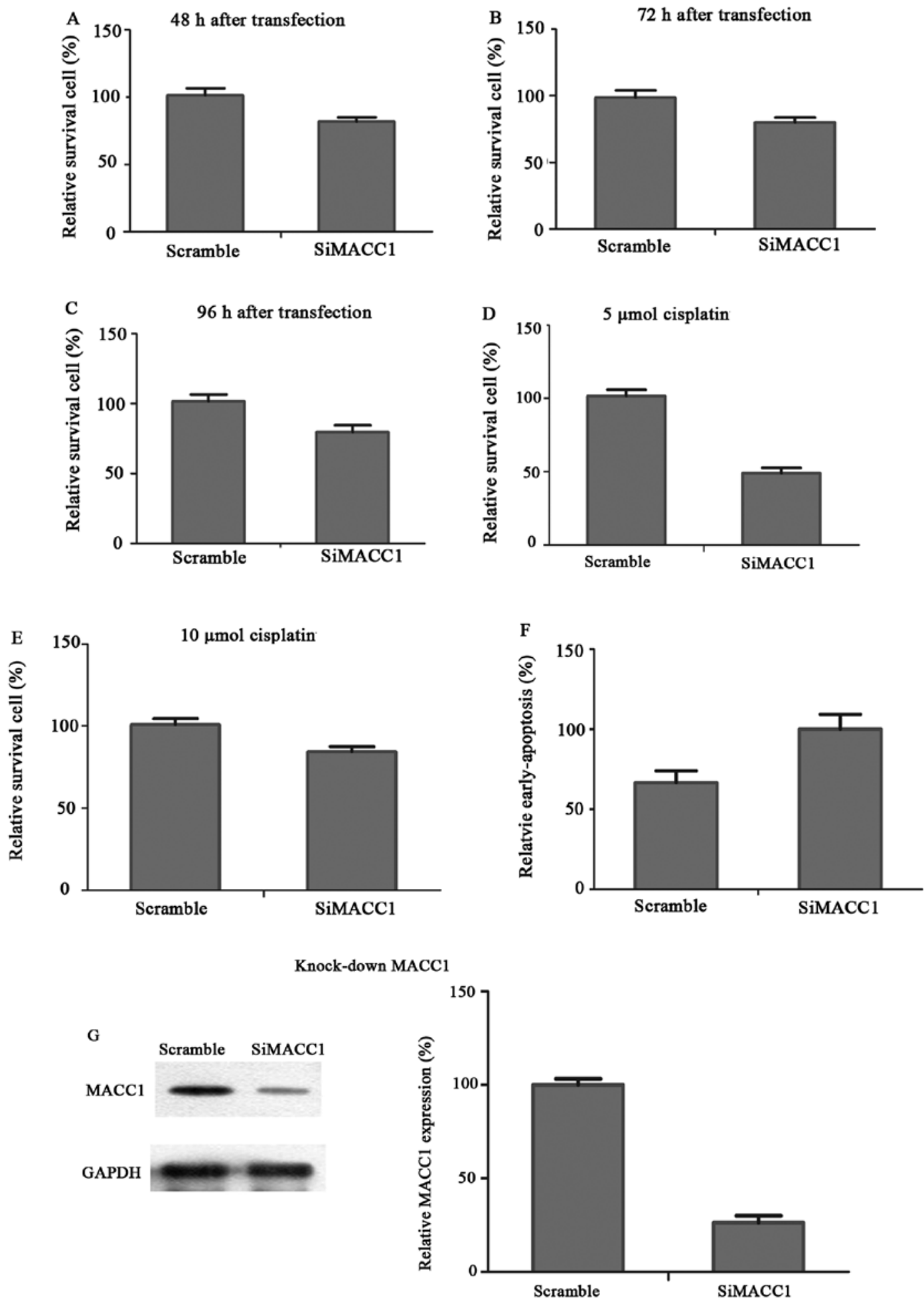

Figure 3. Silencing of MACC1 significantly inhibits proliferation, attenuates cisplatin resistance and induces apoptosis in TSCCA cells. (A) Silencing of MACC1 significantly inhibited the proliferation of TSCCA cells at $48 \mathrm{~h}$ compared with the scramble control, as determined by CCK-8 test (P<0.05). (B) After $72 \mathrm{~h}$ of MACC1 knockdown, cell proliferation was significantly reduced compared with the scramble control, as determined by CCK-8 test $(\mathrm{P}<0.05)$. $(\mathrm{C}) \mathrm{The}$ downregulation of MACC1 inhibited cell proliferation of TSCCA cells at $96 \mathrm{~h}$ compared with the scramble control, as determined by CCK-8 test (P<0.05). (D) Knockdown of MACC1 sensitized TSCCA cells to cisplatin at a concentration of $5 \mu$ mol compared with the scramble control, as determined by CCK- 8 test $(\mathrm{P}<0.05)$. (E) Suppression of MACC1 expression sensitized the TSCCA cells to cisplatin at a concentration of $10 \mu$ mol compared with the scramble control group by CCK8 test $(\mathrm{P}<0.05)$. (F) The downregulation of MACC1 induced apoptosis in the TSCCA cells as determined by flow cytometric analysis $(\mathrm{P}<0.05)$. (G) The MACC1 protein expression of TSCCA cells transfected with MACC1-siRNA was significantly attenuated compared with the NC group (P<0.05). MACC1, metastasis-associated in colon cancer 1; TSCC, tongue squamous cell carcinoma.

the same trend as the migration assay; the number of invasive cells transfected with MACC1-NC and MACC1-siRNA at $48 \mathrm{~h}$ was $1223 \pm 79$ and $749 \pm 34 /$ field of view, respectively. The experiments were carried out a minimum of 3 times. The 

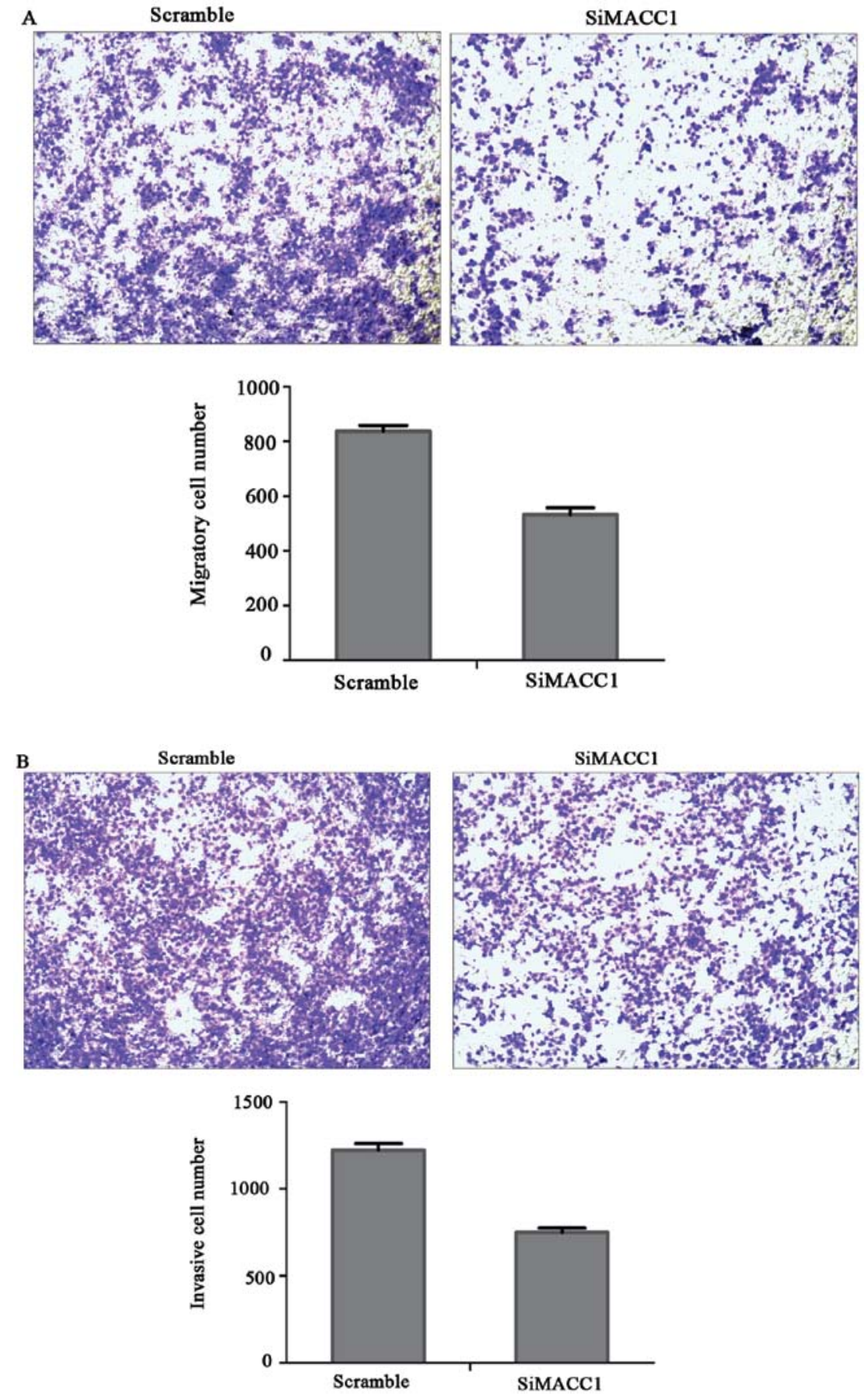

Figure 4. Downregulation of MACC1 contributes to the inhibition of migration and invasion of the TSCCA cell line. (A) Downregulation of MACC1 inhibited the migration of TSCCA cells compared with that of the scramble control, as determined by the Transwell experiment $(\mathrm{P}<0.05)$. (B) The knockdown of MACC1 expression inhibited the invasion of TSCCA cells compared with the scramble control, as determined by the Transwell experiment $(\mathrm{P}<0.05)$. MACC1, metastasis-associated in colon cancer 1; TSCC, tongue squamous cell carcinoma.

differences in the 2 groups (MACC1-siRNA and MACC1-NC) were statistically significant (Fig. $4 \mathrm{~A}$ and $\mathrm{B}, \mathrm{P}<0.05$ ). We conclude that knockdown of MACC1 contributed to the inhibition of migration and invasion of TSCCA cells in vitro.

Knockdown of MACCl markedly reduces the invasive ability through suppression of uPA but not of MMP2 expression and secretion in TSCC in vitro. The ELISA assay demonstrated that the MACC1-siRNA transfection decreased the uPA secretion by nearly $25 \%$. The uPA protein expression was extremely attenuated after transfection with MACC1-siRNA compared with the MACC1-NC group. The difference in UPA secretion and expression between the experimental and the control group was statistically significant (Fig. 5A, B and F, P<0.05). The MMP2 expression analyzed by western blot analysis and the MMP2 secretion detected by ELISA had no significant changes (Fig. 5C, D and G; P>0.05). Based on the results, we primarily concluded that the downregulation of MACC1 inhibited invasion by attenuating uPA rather than by attenuating MMP2 expression and secretion. 

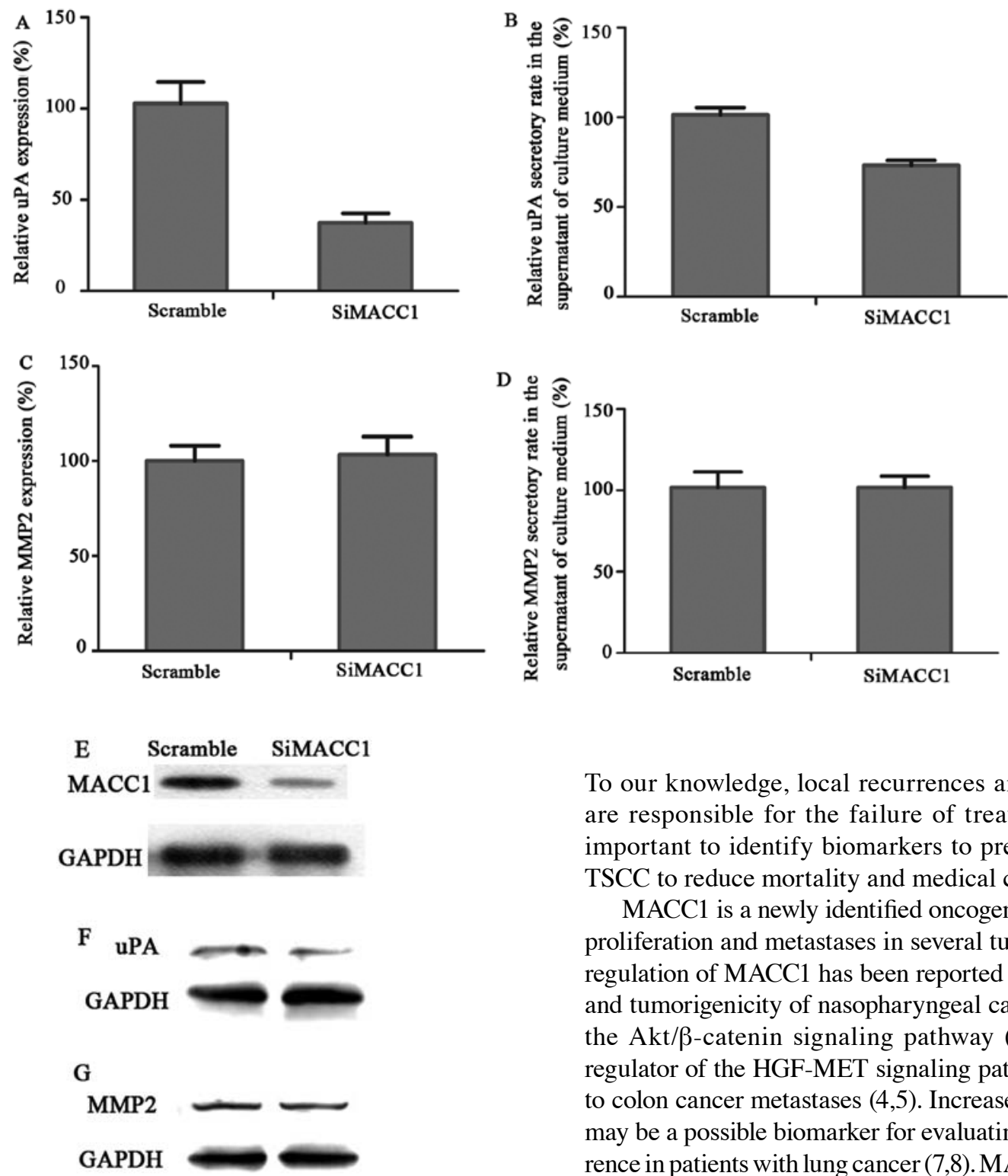

Figure 5. Knockdown of MACC1 markedly reduces the invasive ability of the TSCC A cells by suppressing uPA instead of MMP2 expression and secretion. (A) The knockdown of MACC1 expression reduced uPA protein expression significantly compared to that of the scramble control group by western blot analysis $(\mathrm{P}<0.05)$. (B) The knockdown of MACC1 expression reduced uPA secretion in the supernatant of the culture medium compared with that of the scramble control group by ELISA $(\mathrm{P}<0.05)$. (C) MMP2 expression as analyzed by western blot analysis showed no significant variation after the downregulation of MACC1 expression ( $>00.05$ ). (D) MMP2 secretion as detected by ELISA showed no significant variation after the downregulation of MACC1 expression $(\mathrm{P}>0.05)$. (E) The MACC1 protein expression of TSCCA cells transfected with MACC1-siRNA was significantly attenuated compared with the $\mathrm{NC}$ group by western blot analysis $(\mathrm{P}<0.05)$. (F) The knockdown of MACC1 expression significantly reduced uPA protein expression compared with that of the scramble control group by western blot analysis $(\mathrm{P}<0.05)$. (G) MMP2 expression as analyzed by western blot analysis showed no significant variation after the downregulation of MACC1 expression ( $\mathrm{P}>0.05)$. MACC1, metastasis-associated in colon cancer 1; TSCC, tongue squamous cell carcinoma; uPA, urokinase-type plasminogen activator system.

\section{Discussion}

Although progress has been made in the treatment of TSCC, the prognosis remains poor, with a 5 -year survival rate $<50 \%$ (2).

To our knowledge, local recurrences and distant metastases are responsible for the failure of treatment in TSCC. It is important to identify biomarkers to predict the prognosis of TSCC to reduce mortality and medical costs.

MACC1 is a newly identified oncogene related to malignant proliferation and metastases in several tumor types. The downregulation of MACC1 has been reported to inhibit proliferation and tumorigenicity of nasopharyngeal carcinoma cells through the $A k t / \beta$-catenin signaling pathway (6). MACC1 is a key regulator of the HGF-MET signaling pathway, which is related to colon cancer metastases $(4,5)$. Increased MACC1 expression may be a possible biomarker for evaluating postoperative recurrence in patients with lung cancer $(7,8)$. MACC1 is involved in the progression of breast cancer and represents a potentially useful biomarker for prognosis (14). In other cancers, such as colon carcinoma (4,5), pancreatic cancer (13), ovarian cancer (12) and hepatocellular carcinoma (9-11), MACC1 has been shown to be a new oncogene associated with the malignant biological behaviors of tumors. Whether or not MACC1 expression is related to TSCC has not been previously elucidated. This study determined that MACC1 expression in TSCC was significantly higher than that in the non-cancerous epithelium adjacent to the carcinomas of tongue. Increased MACC1 expression was highly associated with lymphatic metastases and EMMPRIN expression and predicted a poor overall survival of the TSCC patients. Silencing of the MACC1 gene downregulated cell proliferation, migration and invasion and it attenuated the cisplatin resistance of TSCCA cells and contributed to apoptosis. MACC1 influenced the invasion and migration of TSCCA cells by secreting and activating uPA instead of MMP2.

Previous researchers found MACC1 overexpression in various tumor tissues $(6,10,12)$ and pancreatic cancer patient serum samples (13). In nasopharyngeal carcinoma, high MACC1 expression was significantly related to UICC stage $(\mathrm{P}=0.005)$ and $\mathrm{N}$ classification $(\mathrm{P}<0.05)(6)$. In pancreatic cancer patients, a high serum level of MACC1 expression was 
positively correlated with lymph node metastases $(\mathrm{P}=0.035)$, distant metastases $(\mathrm{P}=0.005)$ and more advanced TNM stages $(\mathrm{P}=0.024)$ (13). In our study, we examined 68 TSCC tissues and the non-cancerous epithelial tissues adjacent to the TSCC using IHC. We found that MACC1 expression in TSCC was significantly higher than that in the non-cancerous epithelium adjacent to the TSCC. We analyzed the relationship between clinical characteristics of the patients and MACC1 expression. We discovered that high MACC1 expression was correlated with lymphatic metastases. The multivariate analysis revealed that MACC1 was significantly associated with the overall survival time of TSCC patients. High MACC1 expression contributed to a shorter survival time in TSCC patients. We did not find a significant difference between MACC1 expression and the clinical tumor stage (Table $\mathrm{I}, \mathrm{P}=0.088$ ), but the data did demonstrate a positive trend. High MACC1 expression tended to occur in later stages (III+IV). We suggest that the unclear relationship between high expression of MACC1 and clinical stage could be resolved if we include more cases in our further studies. Researchers have reported that RNA interference against MACC1 resulted in antitumor proliferation and progression in ovarian carcinoma. MACC1 knockdown may be involved in the inhibition of the HGF/MET and MEK/ERK pathways (12). Studies have proposed that the downregulation of MACC1 inhibits the proliferation and tumorigenicity of NCC through the Akt/ $\beta$-catenin signaling pathway (6). The regulation of the Ras/ERK pathway by MACC1 in pancreatic cancer affects metastasis and progression (13). The expression of MACC1 is associated with colon cancer tumorigenesis and metastasis by regulating the HGF/Met signaling pathway $(4,16)$. The mechanism of MACC1 regulation of TSCC remains unclear. To explore the role of MACC1 in TSCC, we employed siRNA to downregulate MACC1 expression and to determine its effect on proliferation, migration, invasion, chemoresistance and apoptosis. A CCK-8 test on TSCCA cells showed that the proliferation was markedly inhibited and that apoptosis was induced after downregulation of MACC1 expression. TSCCA cell sensitivity to cisplatin increased after silencing of the MACC1 gene. The Transwell experiment demonstrated that migration and invasion abilities of the TSCCA cell line were significantly downregulated after transfection with MACC1-siRNA. The results of this study are consistent with the results of previous studies $(6,12)$. We suggest that regulation, proliferation, apoptosis and chemoresistance by MACC1 may involve the HGF/MET, MEK/ERK, Akt/ $\beta$-catenin or Ras/ERK pathway in TSCC. However, the actual mechanism requires further investigation.

Previous studies have demonstrated that EMMPRIN which is a plasma membrane protein of the immunoglobulin (Ig) superfamily, enhances cell proliferation, invasion and metastasis of tumors and contributes to poor overall survival $(18,19)$. In our previous study, EMMPRIN expression, especially EMMPRIN isoform 2 expression, was associated with the metastases of head and neck cancer (20).Overexpression of EMMPRIN promoted proliferation, invasion and metastases of cancer, resulting in poor overall survival $(19,21)$. EMMPRIN expression was significantly associated with tumor diameter, clinical stage and poor survival time in TSCC (2). MACC1 was correlated with carcinoma progression and metastases. It was previously not known whether there is a relationship between MACC1 expression and EMMPRIN expression in TSCC. This study demonstrated that increased MACC1 expression was positively associated with EMMPRIN expression $(\mathrm{P}=0.003)$. We suggest that MACC1 and EMMPRIN may cooperate in carcinogenesis in TSCC. The underlying regulatory mechanism between these genes remains to be further elucidated. Clinically, the failure of treatment in TSCC is due to recurrence and distant metastasis, which seriously affects the prognosis of patients. MACC1 has been identified as an oncogene that is correlated with the metastasis of malignant tumors. The underlying mechanisms involved in the invasion and metastasis of tongue tumors remain unknown. The activation of MMPs, particularly MMP2, has been reported to upregulate invasion and metastases in several malignant carcinomas (22-24). The activation of MMP2 is an important factor mediating invasion in tongue squamous carcinomas $(25,26)$. EMMPRIN stimulates the activation and expression of MMP2, which results in the promotion of invasion (27-29). We previously demonstrated that EMMPRIN activates MMP2 and uPA to enhance invasion in TSCC (20). The uPA has been shown to be closely associated with invasion and metastases of carcinomas (29-32). IHC revealed that MACC1 and EMMPRIN are correlated in predicting malignancy and poor progression. Since EMMPRIN was involved in tumor malignancy and progression, we hypothesized that MACC1 promotes metastasis through the expression and secretion of MMP2 and/or uPA. We used western blot analysis to examine the MMP2 and uPA expression levels. We used ELISA to detect the secretion of MMP2 and UPA in the supernatant of the culture medium. The results showed that uPA expression and secretion were significantly reduced after the knockdown of MACC1 in TSCC cells. No significant variation was found in the MMP2 expression tested by western blot analysis and in the MMP2 secretion detected by ELISA. Therefore, we primarily suggest that the downregulation of MACC1 inhibited invasion by attenuating UPA instead of MMP2 expression and secretion. The actual mechanism needs further investigation.

In conclusion, MACC1 overexpression contributes to metastasis and poor overall survival in TSCC patients. The downregulation of MACC1 inhibits invasion, migration and proliferation, attenuates cisplatin resistance and induces apoptosis in TSCC. MACC1 downregulates the invasion of TSCC by attenuating UPA instead of MMP2 expression and secretion. MACC1 may serve as a novel biomarker and therapeutic target for TSCC.

\section{Acknowledgements}

This study was supported by the Fundamental Research Funds for the Central Universities (no. 13ykpy26), the Guangdong Province Natural Science Foundation (no. S2013010014794) and the National Natural Science Fund of China (81101592).

\section{References}

1. Amar A, Rapoport A, Curioni OA, et al: Prognostic value of regional metastasis in squamous cell carcinoma of the tongue and floor of mouth. Braz J Otorhinolaryngol 79: 734-737, 2013 (In English, and Portuguese).

2. Huang Z, Huang $\mathrm{H}$, Li H, et al: EMMPRIN expression in tongue squamous cell carcinoma. J Oral Pathol Med 38: 518-523, 2009. 
3. Qu JH, Chang XJ,Lu YY, et al: Overexpression of metastasis-associated in colon cancer 1 predicts a poor outcome of hepatitis B virus-related hepatocellular carcinoma. World J Gastroenterol 18: 2995-3003, 2012.

4. Stein U, Burock S, Herrmann P, et al: Circulating MACC1 transcripts in colorectal cancer patient plasma predict metastasis and prognosis. PLoS One 7: e49249, 2012.

5. Stein U, Walther W, Arlt F, et al: MACC1, a newly identified key regulator of HGF-MET signaling, predicts colon cancer metastasis. Nat Med 15: 59-67, 2009.

6. Meng FJ, Li H, Shi HJ, et al: MACCl down-regulation inhibits proliferation and tumourigenicity of nasopharyngeal carcinoma cells through $\mathrm{Akt} / \beta$-catenin signaling pathway. PLoS One 8 : e60821, 2013.

7. Shimokawa H, Uramoto H, Onitsuka T, et al: Overexpression of MACC1 mRNA in lung adenocarcinoma is associated with postoperative recurrence. J Thorac Cardiovasc Surg 141: 895-898, 2011

8. Chundong G, Uramoto H, Onitsuka T, et al: Molecular diagnosis of MACC1 status in lung adenocarcinoma by immunohistochemical analysis. Anticancer Res 31: 1141-1145, 2011.

9. Xie C, Wu J, Yun J, et al: MACC1 as a prognostic biomarker for early-stage and AFP-normal hepatocellular carcinoma. PLoS One 8: e64235, 2013.

10. Qiu J, Huang P, Liu Q, et al: Identification of MACC1 as a nove prognostic marker in hepatocellular carcinoma. J Transl Med 9: 166, 2011.

11. Yang YP, Qu JH, Chang XJ, et al: High intratumoral metastasisassociated in colon cancer-1 expression predicts poor outcomes of cryoablation therapy for advanced hepatocellular carcinoma. J Transl Med 11: 41, 2013.

12. Zhang R, Shi H, Chen Z, et al: Effects of metastasis-associated in colon cancer 1 inhibition by small hairpin RNA on ovarian carcinoma OVCAR-3 cells. J Exp Clin Cancer Res 30: 83, 2011.

13. Wang G, Kang MX, Lu WJ, et al: MACC1: A potential molecule associated with pancreatic cancer metastasis and chemoresistance. Oncol Lett 4: 783-791, 2012.

14. Huang Y, Zhang H, Cai J, et al: Overexpression of MACC1 and its significance in human breast cancer progression. Cell Biosci 3: 16, 2013.

15. Zhang Y, Wang Z, Chen M, et al: MicroRNA-143 targets MACC1 to inhibit cell invasion and migration in colorectal cancer. Mol Cancer 11: 23, 2012

16. Migliore C, Martin V, Leoni VP, et al: MiR-1 downregulation cooperates with MACC1 in promoting MET overexpression in human colon cancer. Clin Cancer Res 18: 737-747, 2012.

17. Guo T, Yang J, Yao J, Zhang Y, Da M and Duan Y: Expression of MACC1 and c-Met in human gastric cancer and its clinical significance. Cancer Cell Int 13: 121, 2013.

18. Zheng HC, Takahashi H, Murai Y, et al: Upregulated EMMPRIN/ CD147 might contribute to growth and angiogenesis of gastric carcinoma: a good marker for local invasion and prognosis. Br J Cancer 95: 1371-1378, 2006.
19. Vigneswaran N, Beckers S, Waigel S, et al: Increased EMMPRIN (CD 147) expression during oral carcinogenesis. Exp Mol Pathol 80: 147-159, 2006.

20. Huang Z, Tan N, Guo W, et al: Overexpression of EMMPRIN isoform 2 is associated with head and neck cancer metastasis. PLoS One 9: e91596, 2014.

21. Zhong WD, Han ZD, He HC, et al: CD147, MMP-1, MMP-2 and MMP-9 protein expression as significant prognostic factors in human prostate cancer. Oncology 75: 230-236, 2008

22. Zheng PC, Chen X, Zhu HW, et al: Capn4 is a marker of poor clinical outcomes and promotes nasopharyngeal carcinoma metastasis via nuclear factor- $\kappa \mathrm{B}$-induced matrix metalloproteinase 2 expression. Cancer Sci 105: 630-638, 2014.

23. Huang Q, Lan F, Wang X, et al: IL-1 $\beta$-induced activation of p38 promotes metastasis in gastric adenocarcinoma via upregulation of AP-1/c-fos, MMP2 and MMP9. Mol Cancer 13: 18, 2014.

24. Moroz A, Delella FK, Almeida R, et al: Finasteride inhibits human prostate cancer cell invasion through MMP2 and MMP9 downregulation. PLoS One 8: e84757, 2013.

25. Xiao W, Jiang M, Li H, Li C, Su R and Huang K: Knockdown of FAK inhibits the invasion and metastasis of Tca8113 cells in vitro. Mol Med Rep 8: 703-707, 2013.

26 . Wang X, Sun W, Zhang C, et al: TGF- $\beta 1$ inhibits the growth and metastasis of tongue squamous carcinoma cells through Smad4. Gene 485: 160-166, 2011.

27. Zucker S, Hymowitz M, Rollo EE, et al: Tumorigenic potential of extracellular matrix metalloproteinase inducer. Am J Pathol 158: 1921-1928, 2001

28. Sienel W, Polzer B, Elshawi K, et al: Cellular localization of EMMPRIN predicts prognosis of patients with operable lung adenocarcinoma independent from MMP-2 and MMP-9. Mod Pathol 21: 1130-1138, 2008 .

29. Bao YN, Cao X, Luo DH, et al: Urokinase-type plasminogen activator receptor signaling is critical in nasopharyngeal carcinoma cell growth and metastasis. Cell Cycle 13: 1958-1969, 2014

30. Shi H, Liu L, Liu L, Geng J, et al: beta-Elemene inhibits the metastasis of B16F10 melanoma cells by downregulation of the expression of uPA, uPAR, MMP-2 and MMP-9. Melanoma Res 24: 99-107, 2014.

31. Quemener C, Gabison EE, Naimi B, et al: Extracellular matrix metalloproteinase inducer up-regulates the urokinase-type plasminogen activator system promoting tumor cell invasion. Cancer Res 67: 9-15, 2007.

32. Lescaille G, Menashi S, Cavelier-Balloy B, et al: EMMPRIN/ CD147 up-regulates urokinase-type plasminogen activator: implications in oral tumor progression. BMC Cancer 12: 115, 2012. 